\title{
System Identification of Constructed Facilities: Challenges and Opportunities Across Hazards
}

\author{
Authors: \\ Tracy Kijewski-Correa, University of Notre Dame, Notre Dame, IN, tkijewsk@nd.edu \\ Ertugrul Taciroglu, University of California, Los Angeles, CA, etacir@ucla.edu \\ James L. Beck, California Institute of Technology, Pasadena, CA, jimbeck@caltech.edu
}

\begin{abstract}
The motivation, success and prevalence of full-scale monitoring of constructed buildings vary considerably across the hazard of concern (earthquakes, strong winds, etc.), due in part to various fiscal and life safety motivators. Yet while the challenges of successful deployment and operation of large-scale monitoring initiatives are significant, they are perhaps dwarfed by the challenges of data management, interrogation and ultimately system identification. Practical constraints on everything from sensor density to the availability of measured input has driven the development of a wide array of system identification and damage detection techniques, which in many cases become hazard-specific. In this study, the authors share their experiences in fullscale monitoring of buildings across hazards and the associated challenges of system identification. The study will conclude with a brief agenda for next generation research in the area of system identification of constructed facilities.
\end{abstract}

\section{INTRODUCTION}

The monitoring of constructed facilities has historically been tied to the need to understand insitu behaviors, often spurred by suspect performance, as in the case of the infamous Boston Hancock Tower. The prevalence of full-scale monitoring in subsequent decades was largely tied, at least in Asia, to the proliferation of auxiliary damping devices, where sensors served a variety of purposes from actual feedback mechanisms in active control to a means to document the performance of these supplementary devices during earthquakes and typhoons. In parallel, monitoring efforts in seismically active zones within the United States received similar emphasis at this time for enhanced understanding of overall behavior and performance, while the wind engineering community turned its full-scale efforts toward developing databases of in-situ dynamic properties. Today, while monitoring efforts worldwide continue the task of in-situ validation of dynamic properties, dynamic load effects and response characteristics, added emphasis on rapid assessment and evaluation in the larger venue of structural health monitoring (SHM) has significantly expanded the literature on system identification (SI), though applications to actual structures are still quite limited.

One obvious goal of most monitoring efforts is the identification of in-situ dynamic characteristics such as periods of vibration, mode shapes and critical damping ratios or in some cases, the direct stiffness and damping matrices themselves. A diverse cross section of approaches has been formulated to achieve these objectives, which can be broadly categorized by the hazard they address (earthquakes vs. strong winds). Thus it is worthwhile to underscore the 
differences between these hazards and the communities addressing them before discussing specific system identification approaches that have been applied in the field.

Table 1 offers a very general overview of the unique objectives and driving forces for monitoring programs focused on seismic and wind/ambient excitations. Due to the spatially and temporally distributed nature of wind pressures in particular, exact measured inputs are never available for system identification, which sharply contrasts with the common situation in seismic zones, although those inputs are nonstationary. Another considerable contrast is the primary limit state of concern, which first and foremost is survival in earthquakes, though performance based engineering certainly has shifted focus to more frequently occurring events. In wind-resistant design, the limit state which usually governs in dynamically-sensitive structures is habitability or accelerations negatively impacting occupant comfort, which relies heavily on the level of viscous damping in the system. As such, the applications in wind-hazard monitoring inevitably shift toward ascertaining accurate equivalent viscous damping ratios for use in linear analyses, whereas, seismic applications may have a greater focus on nonlinear modeling of hysteretic features. Clearly, given the concern for life safety and the considerable cost associated with the inspection and reoccupation process, there are greater fiscal incentives for monitoring in seismic zones, including municipally-sponsored efforts such as the California Strong Motion Instrumentation Program (CSMIP). This leads arguably to a larger number of instrumented buildings, with greater sensor density and even public disclosure of not only the building's identity but also the data itself [NEES News, 2007]. This is in stark contrast to the situation outside of seismic zones in the United States, where an instrumented building is still regarded as a "troubled building" and publicly disclosed monitoring efforts may generate severe public misconception and even liability issues [Kijewski-Correa and Kareem, 2007].

Recently, Kijewski-Correa and Cycon [2007] overviewed the some of the system identification methods that have been applied to actual full-scale data from over thirty individual buildings and three international databases. This study will build on these efforts by providing a more detailed discussion of specific applications to buildings subjected to winds or earthquakes.

\begin{tabular}{lll}
\hline Feature & Seismic & Wind/Ambient \\
\hline Input & Measured at the base, & Unknown, Spatially/Temporally \\
& Nonstationary & Distributed, Broadly Stationary \\
Response Type & Dynamic & Static, Quasi-Static, Dynamic \\
Primary Limit State & Survivability & Serviceability, Habitability \\
Model & Linear and Nonlinear, Hysteretic & Linear, Equivalent Viscous \\
Sensor Density & Comparatively Higher & Generally Low \\
Incentives & Life Safety, Performance Based & Scarce (Performance Evaluation?) \\
& Engineering, Rapid Reoccupation & \\
\hline
\end{tabular}

TABLE 1 - COMPARISON OF SEISMIC AND WIND/AMBIENT MONITORING EFFORTS

\section{SEISMIC APPLiCATIONS}

Earthquake engineers have instrumented many structures to record their motion during earthquakes in order to quantitatively assess earthquake-resistant design practices and to provide information to improve these practices; e.g., one of the largest instrumentation programs is the CSMIP of the California Geological Survey (http://www.conservation.ca.gov/CGS/SMIP/), who have accelerometer arrays distributed over many buildings, bridges and dams, in addition to ground motion stations, throughout California. Despite the large number of structural strong- 
motion records that have been obtained worldwide and their importance to the earthquake engineering profession, the analysis of these data has not become routine and only a small fraction of the available data has been published, mostly as a result of research studies. Most of these analyses perform modal identification, where parameters of the lower modes of vibration are identified. Here, we focus on this application.

Modal identification applied to recorded seismic response is a rather mature area of system identification research. Most of the studies have estimated the modal parameters (natural frequencies, modal damping ratios, modal input participation factors, and mode shape components at the observed degrees of freedom) by using a non-linear optimization to perform time-domain least-squares matching of the measured and model responses based on a model consisting of the superposition of the lower modes of vibration. Early theory and applications are in Beck [1978], Beck and Jennings [1980] and, for multi-input multi-output, in Werner et al. [1987]; (see also, the Theory section at the website for the Caltech Online Monitoring and Evaluation Testbeds, http://comet.caltech.edu/). The least-squares output-error matching can also be performed in the frequency domain using the complex Fourier transforms of the data and model outputs [McVerry, 1980]; the time-domain and frequency-domain versions are essentially equivalent because of Parseval's identity. Usually the modal models are based on uncoupled classical modes but least-squares matching based on non-classical modes of vibration can also be used [Tan and Cheng, 1993].

Past studies of data recorded in mid-rise buildings during earthquakes [e.g., Beck, 1978; Beck and Jennings, 1980; McVerry, 1980; McVerry and Beck, 1983; Papageorgiou and Lin, 1989a,b; Li and Mau, 1991; Nisar et al., 1992; Hashimoto et al., 1993; Durrani et al., 1994; Mau and Aruna, 1994] have revealed that a small number of modes (5 to 10) can capture the translational and torsional behavior of tall buildings during non-damaging earthquakes. Identified modal periods are typically within $10 \%$ to $15 \%$ of the corresponding values calculated from theoretical finite-element models (FEMs). These periods differ from values given by simple code-based formulas by much larger amounts, and they greatly exceed the periods identified from small-amplitude ambient vibration tests. For non-damaging response, identified equivalent viscous damping ratios for each mode are typically in the range 3\% to $8 \%$ of critical damping.

This summary of results refers to equivalent linear modal parameters from "time-invariant" models of a structure, where the entire duration of the recorded earthquake motions is used. In addition, the time variation of these parameters has been studied by using smaller time windows of data (two fundamental periods or more in length). This procedure can provide insight into the extent and nature of the nonlinearities in the structural behavior. In particular, many of the above studies have shown that substantial stiffness losses of the order of $50 \%$ or more can occur as the strong-motion amplitudes build up during the earthquake, even if the structure is undamaged and has not undergone significant plastic yielding. This stiffness loss may be due to one or more sources: micro-cracking of concrete, loosening of connections between nonstructural components and the principal structural load-carrying system, changes in boundary conditions such as softening in the soil foundations, and other effects. Most of the stiffness loss is recovered as the motion subsides. Studies to localize these stiffness losses are of much interest but appear to require more dense instrumentation arrays than the 10 to 20 or so accelerometers that are typically distributed over buildings at present.

The estimated prediction-error standard deviations, which are given by the square root of the mean-square output-error for the optimal identified modal model, range from about $15 \%$ to $70 \%$ of the root-mean-square of the measured accelerations, with the low end corresponding to small 
non-damaging seismic motions and the high end corresponding to damaging response where "time-invariant" linear dynamic models do not capture well the enormous changes in stiffness that occur. A better class of models could be developed which include a dependence of the prediction-error variance on the strength of shaking; identification of such models could be performed using data for different strengths of shaking recorded in the same building, e.g. some buildings in San Jose, California, have had their motion measured during multiple earthquakes, which produced substantially different peak horizontal roof accelerations; in one case, the range was from $8 \%$ to $38 \%$ g [Nisar et al., 1992].

The modal identification approach has also been used to determine the level of earthquakeinduced forces, base shears, overturning moments and deformations that are then compared with code design values [e.g., Nisar et al., 1992; Mau and Aruna, 1994]. This is done by producing complete mode shapes from the identified components through some form of simple "interpolation"; e.g., fitting a curve through the known floor mode shape components to estimate the components at the un-instrumented floors. A conventional modal time-history analysis can then be performed using the recorded base motions as input because a complete set of modal parameters is available for those modes contributing significantly to the building's seismic response. Such a study [Nisar et al., 1992] has shown that earthquake-induced base shears and overturning moments can exceed design levels by factors of 2 to 3 without any structural damage, confirming that structures designed to code levels often have considerable overstrength. The same study of recorded non-damaging seismic response confirms that the interstory drift ratios correlate better with observed seismic performance since the seismically-induced drift ratios were no more than a few tenths of a percent, even when one building experienced a peak horizontal roof acceleration of 38\% $g$ during the 1989 Loma Prieta earthquake in California.

\section{Wind/AMbient VibRATION APPLICATIONS}

As discussed in Kijewski-Correa and Cycon [2007], both in the monitoring of individual tall buildings under wind and in the development of databases, the primary objectives have been determining the accuracy of predicted response quantities from wind tunnel test data (static, quasi-static and dynamic RMS displacements and RMS accelerations) and the in-situ dynamic properties for validation of FEM assumptions affecting frequency and determination of realistic viscous damping levels for various structural systems. In fact, it may be argued that accurate viscous damping identification is one of the major needs within this community. Here sensor arrays are comprised generally of 2-6 accelerometers/1-2 global positioning antennas only on one of the uppermost floors/roof. Given that measured input is lacking, system identification is resigned to output-only methodologies reliant on the assumption of white noise driving a linear system yielding stationary, ergodic responses. As a result, a significant amount of data is required to estimate power spectral densities or autocorrelation functions with any accuracy. The inherent trade offs between bias and random errors in power spectral estimates have prompted a recent shift toward time-domain methodologies. For example, correlation functions have been fed into the Eigensystem Realization Method to extract the dynamic properties of a building at Saitama University [Areemit et al., 2003]. This study underscored the importance of sufficient data to accurately estimate the correlation function, as well as to form a Hankel matrix of sufficient dimension. As commonly observed in other ambient vibration investigations, frequencies were repeatably estimated, though damping and mode shapes showed poor stabilization and were thus deemed unreliable. Similar experiences with direct determination of correlation functions has helped propel the random decrement technique (RDT) as an alternative 


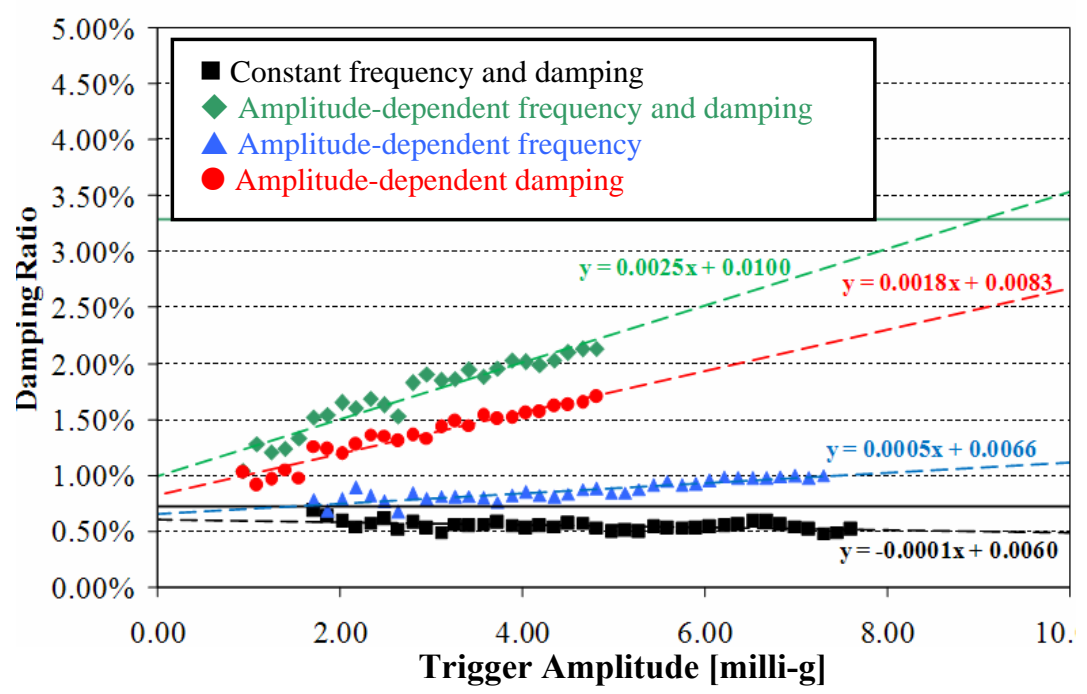

FIGURE 1 - PERFORMANCE OF RANDOM DECREMENT TECHNIQUE IN ESTIMATING AMPLITUDE-DEPENDENT DYNAMIC PROPERTIES OF TALL BUILDINGS

for autocorrelation estimation. The primary advantages in this case are the ability to relax stationarity requirements and capture mild nonlinearities in frequency and damping [Tamura and Suganuma, 1996]. To maintain faithfulness to the high-frequency base balance methodologies common to design practice, linear models are maintained in wind-resistant design and frequency and damping values used in these evaluations are then adjusted for events of varying return period to account for their amplitude dependence. Thus the identification of "amplitudedependent" dynamic properties of tall buildings has become quite common, as seen in the case of the Di Wang Tower, Central Plaza Tower, Bank of China, and an anonymous collection of tall buildings in Chicago and Seoul [Li et al., 2005; 2003; Kijewski-Correa and Pirnia, 2007]. The wider program the latter study was a part of further verified the validity of common FEM assumptions for cantilever-dominated steel structures. However, reinforced concrete interactive systems were found to be stiffer in-situ (by up to $25 \%$ ), which may be attributed to differences in in-situ modulus of elasticity or model stiffness reductions due to cracking that has yet to be observed in the service life of the building. Conversely, interactive steel systems deviated from FEM predictions, yielding longer in-situ periods by approximately $10 \%$, potentially due to unmodeled panel zone deformations [Kijewski-Correa et al., 2006]. These full-scale investigations have also formulated hypotheses surrounding energy dissipation capabilities tied to dominant deformation mechanisms in these systems [Bentz and Kijewski-Correa, 2008].

Recent RDT studies utilized a vector of target amplitude triggers to generate suites of damping and frequency estimates tied to a narrow amplitude range. This form of local averaging has been shown to improve RDT performance by minimizing trigger sensitivity, while still permitting documentation of the degree of amplitude dependence in viscous damping ratios of several tall buildings and the effect of amplitude-dependent frequency on not only equivalent viscous damping, as shown in Figure 1, but also as a facilitator of significant lateral-torsional beat phenomena at modest wind speeds [Kijewski-Correa and Pirnia, 2007].

As evidenced by the Japanese Damping Database [Satake et al., 2003], approximately half the full-scale applications for wind or ambient vibration monitoring still employ frequency domain identification techniques, such as frequency domain decomposition [e.g., Rainieri et al., 


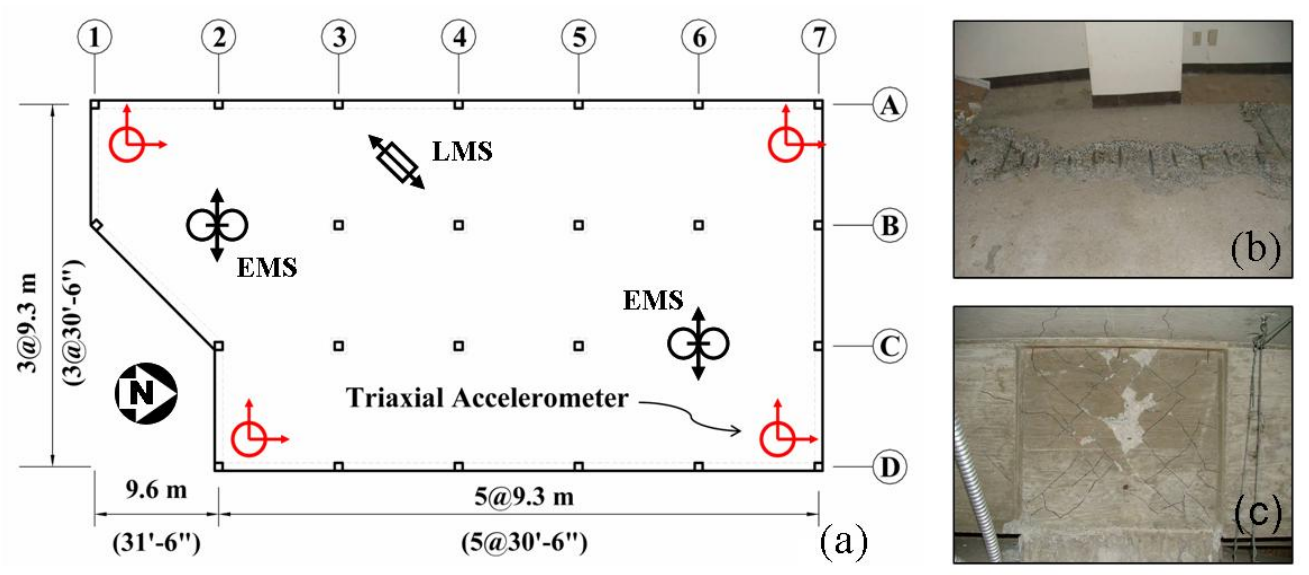

FIGURE 2 - THE FOUR SEASONS BUILDING (A) TYPICAL FLOOR PLAN SHOWING SENSOR AND SHAKER LOCATIONS, OBSERVED DAMAGE DUE TO (B) SLAB PUNCHING AND (C) JOINT SHEAR CRACKING

2007] or half power bandwidth [e.g., Campbell et al., 2005]. In the former application, the singular values meeting minimum modal assurance criteria were transformed back to the time domain through inverse Fourier transform to permit logarithmic estimates of damping. Still, there is significant reluctance to use frequency domain methods due to the many signal processing issues associated with fast Fourier transforms and the inclusion of windows that inflate damping estimates. In particular, given the increased variance that often results when low bias spectra are generated, methodologies like maximum likelihood estimators have been employed and were shown by Erwin et al. [2007] to produce lower bias and variance in their damping estimates than traditional methods applied to high variance power spectra from a collection of buildings in South Korea.

\section{From System Identification to Damage ASSESSMENT}

Inevitably, the field of system identification has expanded to include a wide class of vibrationbased damage detection methods. Unfortunately, the field of structural health monitoring is still very much in its infancy with respect to successful full-scale deployments in buildings, leaving few examples to offer here. Those that have been attempted to date have had mixed success. For example, Turek and Ventura [2007] applied the Damage Locating Vector (DLV) technique, a flexibility-based method [Bernal and Gunes, 2004], which was adapted to this ambient vibration analysis using approximate flexibilities by the Stochastic Damage Locating Vector (SDLV) [Bernal, 2006] and the Proportional Flexibility Matrix (PFM) [Duan et al., 2005] techniques. These approaches were employed on the Melville Building and the Heritage Court Tower, respective 44- and 15-story reinforced concrete buildings in Vancouver. This study highlighted a major practical limitation in flexibility-based identification: the difficulty in achieving a sufficient sensor density to resolve even a simplified frame representation of the inherently more complex actual structure. Even more challenging is the fact that in output-only identification only approximate mode shapes can be obtained, leading to a failure of the DLV in the field.

On the other hand, careful application of some well-known techniques, albeit with certain critical enhancements, has yielded practically useful results in the area of damage assessment. One such study has been performed on the Four Seasons Building located in Sherman Oaks, California, which is a four-story reinforced concrete special moment frame that was damaged 
during the 1994 Northridge earthquake [Yu et al., 2007a,b]. Slated for demolition, it remains unoccupied since that incident. Figure 2a shows the typical floor plan and sensor layout. Visual inspections were performed to document the earthquake damage, which included slab punching failures (Fig. 2b), significant diagonal cracks in beam-column joint regions (Fig. 2c), column flexural cracks, and concrete spalling at beam ends adjacent to the beam-column joints. A series of forced vibration tests were conducted on this building in 2004 using the nees@UCLA equipment (www.nees.ucla.edu/fourseasons.htm), which is comprised of advanced wireless data acquisition systems and linear and eccentric mass shakers (labeled as LMS and EMS on Fig. 2a, respectively). The Numerical algorithm for Subspace State-Space System Identification (N4SID) was adopted in the analysis of this full-scale dataset [Van Overschee and De Moor, 1994]. This non-iterative approach yields reliable state-space models for complex multivariate dynamical systems, directly from measured data with modest computational effort. The modal properties are easily deduced from the back-calculated state-space model and are displayed in Table 2.

\begin{tabular}{|c|c|c|c|c|c|c|c|c|c|}
\hline \multicolumn{2}{|c|}{ Mode } & \multicolumn{2}{|c|}{ Identified } & \multicolumn{3}{|c|}{ Initial Model } & \multicolumn{3}{|c|}{ Updated Model } \\
\hline No & Dir & $f_{m}(\mathbf{H z})$ & $\zeta(\%)$ & $f_{\mathrm{i}}(\mathrm{Hz})$ & $f_{\mathrm{i}} / f_{m}$ & MAC* & $f_{u}(\mathrm{~Hz})$ & $f_{\mathrm{u}} / f_{m}$ & MAC* \\
\hline 1 & EW & 0.88 & 5.66 & 0.89 & 1.01 & 0.98 & 0.89 & 1.01 & 1.00 \\
\hline 2 & NS & 0.94 & 6.94 & 1.08 & 1.15 & 0.99 & 0.96 & 1.02 & 0.99 \\
\hline 3 & Tor & 1.26 & 6.01 & 1.29 & 1.02 & 1.00 & 1.26 & 1.00 & 1.00 \\
\hline 4 & EW & 2.73 & 5.61 & 2.64 & 0.97 & 0.90 & 2.72 & 1.00 & 0.99 \\
\hline 5 & NS & 2.94 & 7.69 & 2.99 & 1.02 & 0.94 & 2.93 & 1.00 & 0.98 \\
\hline 6 & Tor & 3.44 & 6.14 & 3.42 & 0.99 & 0.93 & 3.44 & 1.00 & 0.99 \\
\hline
\end{tabular}

TABLE 2 - Four SeAsons BuILding Modal PRoperties

Subsequent to modal identification, an initial FEM was constructed based on architectural/structural drawings and reasonable assumptions. Even though the initial FEM was constructed with considerable care [Yu et al., 2007b], there were significant discrepancies between these identified and analytical modal properties as shown in Table 2. Model updating was performed to reduce these discrepancies. As it is well known, this second nonlinear inverse problem is inherently ill-conditioned with non-unique solutions, because groups of distinct model parameters may have very similar influences on the error residuals. As such, the updating parameters — which included translational masses, gyration radii, and effective beam, column, slab stiffnesses - had to be grouped to reduce the number of independent unknowns. It was not entirely possible to determine a priori what the (pareto-) optimal grouping of parameters would be, so a novel strategy to adaptively constrain the updating parameters was devised and first verified on model problems [Skolnik et al., 2006]. This enhanced model updating method was effective, convergent, and yielded reasonable results with improved agreement between identified and computed (from updated FEM) modal data, as further shown in Table 2.

Figure 3a displays the good agreement between the updated and measured transfer functions for the Four Seasons Building. Figure 3b shows the initial grouping of the updating parameters that was based on member types and their vertical locations. The aforementioned adaptive constraining algorithm further clustered the updating parameters based on the error function's sensitivity to these groups to yield a convergent solution. A subset of the final values of the updated effective stiffness factors is displayed in Figure 3c. Several of these updated factors 

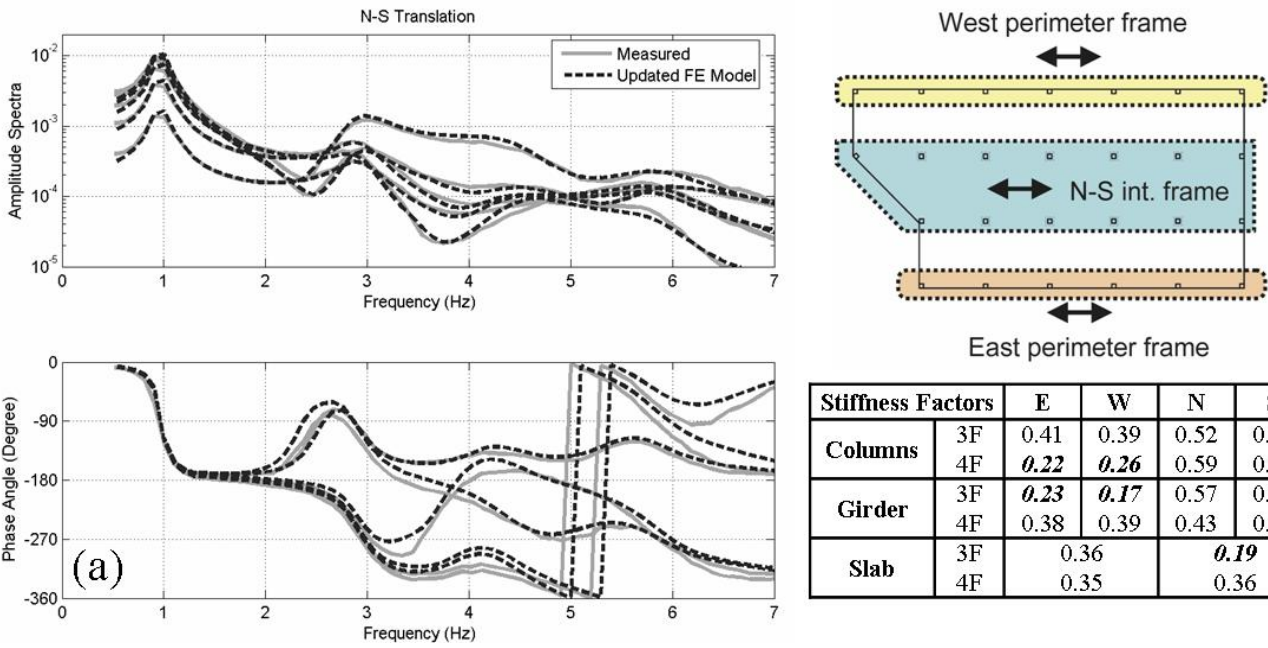

\begin{tabular}{|c|c|c|c|c|c|}
\hline \multicolumn{2}{|c|}{ Stiffness Factors } & E & W & N & S \\
\hline \multirow{2}{*}{ Columns } & $3 \mathrm{~F}$ & 0.41 & 0.39 & 0.52 & 0.56 \\
& $4 \mathrm{~F}$ & $\mathbf{0 . 2 2}$ & $\mathbf{0 . 2 6}$ & 0.59 & 0.34 \\
\hline \multirow{2}{*}{ Girder } & $3 \mathrm{~F}$ & 0.23 & $\boldsymbol{0 . 1 7}$ & 0.57 & 0.32 \\
& $4 \mathrm{~F}$ & 0.38 & 0.39 & 0.43 & 0.36 \\
\hline \multirow{2}{*}{ Slab } & $3 \mathrm{~F}$ & \multicolumn{2}{|c|}{0.36} & \multicolumn{2}{c|}{0.19} \\
& $4 \mathrm{~F}$ & \multicolumn{2}{|c|}{0.35} & \multicolumn{2}{c|}{0.36} \\
\hline
\end{tabular}

FIGURE 3 - FOUR SEASONS BUILDING: (A) COMPARISON OF MEASURED AND PREDICTED TRANSFER FUNCTIONS, (B) SAMPLE DIAGRAM OF INITIAL GROUPING OF UPDATING STIFFNESS PARAMETERS, AND (C) TABLE OF SELECT FINAL EFFECTIVE STIFFNESS FACTORS BASED ON UPDATED PARAMETERS

were significantly reduced from their initial values of 0.5 (indicated with bold-italic numbers in Fig. 3c) and corresponded well to locations of observed damage in the building.

\section{FUtURe Directions}

The previous studies indicate that existing modal identification techniques are reasonably mature and yield realistic results with minimal ad hoc interference by the user. However, the FEM updating is a more difficult problem to solve, and even with enhancements, it requires the user to make a number of critical assumptions as the results ultimately depend upon user-defined weights and constraints. Nevertheless, with due care, it is clearly possible to obtain accurate updated finite element models, and even to quantify damage in the form of stiffness degradation. It may be possible to correlate such data to strength degradation through subsequent computations with reliable models of inelastic component behavior.

State-of-the-art in modal and model updating can be significantly improved with measurements and methods that combine data from different types of sensors. Wellsynchronized measurements of inter-story displacements, slab/beam/column strains and beamcolumn joint rotations may yield more reliable results than those obtained by lateral acceleration measurements alone. Moreover, in most studies to date, including those presented here, the obtained results are actually the mean values of estimated structural parameters. System identification of constructed facilities should look toward methods that provide a framework to quantify the uncertainties of model parameters [e.g., Beck and Katafygiotis, 1998]. Such methods would better reflect the inherent uncertainty in measurements and models for direct incorporation into reliability analyses and may even help determine improved sensor deployment strategies and design networks with increased sensitivity and efficiency [e.g., Papadimitriou, et al., 2000].

As examples herein have also demonstrated, many existing methodologies are constrained by practical limits on sensor density. With the continued movement toward ubiquitous sensing paradigms employing high density wireless sensor networks, one practical constraint on system 
identification (low sensor density) will be lifted as another is imposed - the need to develop embeddable, decentralized approaches to system identification that can be executed using the limited power and computational resources of wireless sensor motes.

\section{ACKNOWLEDGEMENT}

The authors wish to acknowledge their wider affiliation with the ASCE Technical Committee on System Identification of Constructed Systems, the efforts of their collaborators and graduate students, and the support of their sponsors for these system identification programs.

\section{REFERENCES}

[1] Areemit, N., H. Yamaguchi, Y. Matsumoto and T. Ibi, "Model identification of a four story reinforced concrete building under renovation using ambient vibration measurement," Str. Health Monitoring and Intelligent Infrastructure, Wu \& Abe (Eds.), Swets \& Zeitlinger, Lisse, The Netherlands, 2003, 513-520.

[2] Beck, J.L., Determining Models of Structures from Earthquake Records, EERL Report No. 78-01, California Institute of Technology, Pasadena, 1978 (http://caltecheerl.library.caltech.edu/183/).

[3] Beck, J.L. and P.C. Jennings, “Structural identification using linear models and earthquake records”, Earthquake Engr. and Str. Dynamics, 8, 1980, 145-160.

[4] Beck, J.L. and L.S. Katafygiotis, “Updating models and their uncertainties: Bayesian statistical framework”, J. Engr. Mech., Vol. 124, No. 4, 1998, 455-461.

[5] Bentz, A. and T. Kijewski-Correa, "Predictive models for damping in buildings: the role of structural system characteristics”, Proc. of 2008 Str. Cong., 18 ${ }^{\text {th }}$ Analysis \& Comp. Specialty Conf., Vancouver, Canada, 2008.

[6] Bernal, D. and B. Gunes, “Flexibility based approach for damage characterization: benchmark application”, J. Engr. Mech., Vol. 130, 2004, 61-70.

[7] Bernal, D., “Flexibility-based damage localization from stochastic realization results”, J. Engr. Mech., Vol. 132, No. 6, 2006, 651-658

[8] Campbell, S., K.C.S. Kwok and P.A. Hitchcock, "Dynamic characteristics and wind-induced response of two high-rise residential buildings during typhoons”, J. of Wind Engr. and Ind. Aero., Vol. 93, 2005, 461-482.

[9] Duan, Z., Yan, G., Ou, J. and B.F. Spencer, "Damage localization in ambient vibration by constructing proportional flexibility matrix”, J. Sound and Vib., Vol. 284, 2005, 455-466.

[10] Durrani, A.J., S.T. Mau, A.A. AbouHashish and Y. Li, "Earthquake response of flat-slab buildings", J. Str. Engr., Vol.120, 1994, 947-964.

[11] Erwin, S., T. Kijewski-Correa and S.-Y. Yoon, "Full-scale verification of dynamic properties from short duration records”, Proc. ASCE Struct. Cong., Long Beach, CA, May 2007, CD-ROM.

[12] Hashimoto, P.S., L.W. Tiong, L.K. Steele, J.J. Johnson and J.L. Beck, Stiffness and Damping Properties of a Low Aspect Ratio Shear Wall Building Based on Recorded Earthquake Responses, Report NUREG/CR-6012, U.S. Nuclear Regulatory Commission, Washington, D.C., 1993.

[13] Kijewski-Correa, T. and J.D. Pirnia, "Dynamic behavior of tall buildings under wind: insights from full-scale monitoring”, The Struct. Design of Tall and Special Buildings, Vol.16, 2007, 471-486.

[14] Kijewski-Correa, T. and J. Cycon, "System identification of constructed buildings: current state-of-the-art and future directions”, Proc. of SHM-II 2007, November, Vancouver, Canada, 2007, CD-ROM.

[15] Kijewski-Correa, T. and A. Kareem, "Monitoring serviceability limit states in civil infrastructure: lessons learned from the Chicago full-scale monitoring experience," Proc. of 6th Intl. Workshop on Struct. Health Monitoring, September 11-13, 2007, Stanford University.

[16] Kijewski-Correa, T., J. Kilpatrick, A. Kareem, D.K. Kwon, R. Bashor, M. Kochly, B.S. Young, A. Abdelrazaq, J. Galsworthy, N. Isyumov, D. Morrish, R.C. Sinn and W.F. Baker, "Validating the wind-induced response of tall buildings: a synopsis of the Chicago full-scale monitoring program”, J. Str. Engr., Vol. 132, No. 10, 2006, 1509-1523.

[17] Li, Q.S., K. Yang, C.K. Wong, and A.P. Jeary, 'The effect of amplitude-dependent damping on wind-induced vibrations of a super tall building', J. of Wind Engr. and Ind. Aero., Vol. 91, 2003, 1175-1198.

[18] Li, Q.S., Y.Q. Xiao and C.K. Wong, 'Full-scale monitoring of typhoon effects on super tall buildings', J. of Fluids and Struct., Vol. 20, 2005, 697-717. 
[19] Li, Y. and S.T. Mau, "A case study of MIMO system identification applied to building seismic records", Earthquake Engr. and Str. Dyn., Vol. 20, 1991, 1045-1064.

[20] Mau, S.T. and V. Aruna, "Story drift, shear and OTM estimation from building seismic records", J. of Str. Engr., Vol.120, 1994, 3366-3385.

[21] McVerry, G.H., "Structural identification in the frequency domain from earthquake records", Earthquake Engr. and Str. Dyn., Vol. 8, 1980, 161-180.

[22] McVerry, G.H. and J.L. Beck, Structural Identification of JPL Building 180 using Optimally Synchronized Earthquake Records, EERL Report No. 83-01, California Institute of Technology, Pasadena, 1983 (http://caltecheerl.library.caltech.edu/155/).

[23] NEES News, “Turning tall buildings into seismic test beds”, EERI Newsletter, Vol. 41, No. 11, Nov. 2007, 8.

[24] Nisar, A., S.D. Werner and J.L. Beck, "Assessment of UBC seismic design provisions using recorded building motions”, Proc. of $10^{\text {th }}$ World Conf. on Earthquake Engr., Madrid, Spain, 1992.

[25] Papadimitriou, C., J.L. Beck and S.K. Au, "Entropy-based optimal sensor location for structural model updating,” J. Vib. and Control, Vol. 6, 2000.

[26] Papageorgiou, A.S. and B.C. Lin, "Influence of lateral-load-resisting system on the earthquake response of structures - a system identification study. Earthquake Engr. and Str. Dyn., Vol.18, 1989a, 799-814.

[27] Papageorgiou, A.S. and B.C. Lin, "Study of the earthquake response of the base-isolated Law and Justice Center in Rancho Cucamonga”, Earthquake Engr. and Str. Dyn., Vol.18, 1989b, 1189-1200.

[28] Rainieri, C., G. Fabbrocino, G. Manfredi and E. Cosenza, "Structural monitoring and assessment of the school of engineering main building at University of Naples Federico II", Proc. of $3^{\text {rd }}$ Intl. Conf. on Struct. Health Monitoring of Intelligent Infrastructure, Vancouver, November 13-16, 2007, CD-ROM: \#89.

[29] Satake, N., K. Suda, T. Arakawa, A. Sasaki, and Y. Tamura, 'Damping evaluation using full-scale data of buildings in Japan', J. Str. Engr., Vol.129, No. 4, 2003, 470-477.

[30] Tamura, Y. and S.-Y. Suganuma, "Evaluation of amplitude-dependent damping and natural frequency of buildings during strong winds”, J. Wind Engr. and Ind. Aero., Vol. 59, 1996, 115-130.

[31] Tan, R.Y. and W.M. Cheng, "System identification of a non-classically damped linear system”, Computers and Struct., Vol. 46, 1993, 67-75.

[32] Turek, M. and C.E. Ventura, "A method for implementation of damage detection algorithms for civil SHM systems", Proc. of $3^{\text {rd }}$ Intl. Conf. on Struct. Health Monitoring of Intelligent Infrastructure, Vancouver, November 13-16, 2007, CD-ROM: \#89.

[33] Van Overschee, P and B. DeMoor, "N4SID: subspace algorithms for the identification of combined deterministic-stochastic systems”, Automatica, Special Issue on Statistical Signal Processing and Control, Vol. 30, No. 1, 1994, 75-93.

[34] Werner, S.D., J.L. Beck and M.B. Levine, "Seismic response evaluation of Meloland Road Overpass using 1979 Imperial Valley earthquake records”, Earthquake Engr. and Str. Dyn., Vol. 15, 1987, 249-274.

[35] Yu, E., E. Taciroglu and J.W. Wallace, "Parameter identification of framed structures using an improved finite element model updating method, Part I: Formulation and verification; Part II: Application to experimental data”, Earthquake Engr. \& Str. Dyn., Vol. 36, 2007a, 619-639.

[36] Yu, E., J.W. Wallace, and E. Taciroglu "Parameter identification of framed structures using an improved finite element model updating method, Part II: Application to experimental data”, Earthquake Engr. \& Str. Dyn., Vol.36, 2007b, 641-660. 